\title{
Tumores de tronco encefálico: reflexión moral sobre la conducta quirúrgica
}

Adriana Vilma Scrigni ${ }^{1}$, Beatriz Elida Mantese ${ }^{1}$

1. Hospital de Pediatría Prof. Dr. Juan P. Garrahan, Buenos Aires, Argentina.

\section{Resumen}

Los tumores de tronco encefálico representan alrededor del $10 \%$ al $20 \%$ de los tumores del sistema nervioso central en niños. El glioma intrínseco difuso es el más frecuente (80\% de los casos) de este grupo de tumores, que se caracterizan por la mala evolución y una sobrevida corta. El diagnóstico se puede hacer por resonancia magnética (con espectroscopía) o por biopsia estereotáxica, un método controvertido, que permite el estudio inmunohistoquímico y molecular del tumor. La reflexión moral se focaliza en la indicación de biopsia para pacientes vulnerables y con mal pronóstico. Se analiza la cirugía desde el punto de vista ético, con base en el mejor interés del niño y en la actitud altruista del paciente y su familia.

Palabras clave: Neoplasias. Neurocirugía. Biopsia. Bioética. Niño.

\section{Resumo}

Tumores de tronco encefálico: reflexão moral sobre a conduta cirúrgica

Os tumores de tronco encefálico representam cerca de $10 \%$ a $20 \%$ dos tumores do sistema nervoso central em crianças. O glioma intrínseco difuso é o mais frequente (80\% dos casos) desse grupo de tumores, caracterizados pela má evolução e por uma curta sobrevida. O diagnóstico pode ser feito por ressonância magnética (com espectroscopia) ou por biópsia estereotáxica, um método controverso, que permite o estudo imunohistoquímico e molecular do tumor. A reflexão moral se concentra na indicação de biópsia para pacientes vulneráveis e com mau prognóstico. A cirurgia é analisada do ponto de vista ético com base no melhor interesse da criança e na atitude altruísta do paciente e da família.

Palavras-chave: Neoplasias. Neurocirurgia. Biópsia. Bioética. Criança.

\section{Abstract}

Brainstem tumors: moral reflection on surgery management

Brainstem tumors represent about $10 \%$ to $20 \%$ of central nervous system tumors in children. Diffuse intrinsic glioma is the most frequent ( $80 \%$ of cases) in this group of tumors, characterized by poor prognosis and short survival. Diagnosis can be made by magnetic resonance (with spectroscopy) or by stereotactic biopsy, a controversial method that allows immunohistochemical and molecular study of the tumor. Moral reflection focuses on the indication of biopsy for vulnerable patients with a poor prognosis. Surgery is analyzed from an ethical point of view based on the best interests of the child and altruistic attitude of patient and family.

Keywords: Neoplasms. Neurosurgery. Biopsy. Bioethics. Child. 


\section{Dilema bioético}

Los tumores de tronco encefálico representan alrededor del $10 \%$ al $20 \%$ de los tumores del sistema nervioso central en niños, con 40 nuevos casos al año en Gran Bretaña y de 300 a 400 casos en Estados Unidos. En Argentina, en un hospital de alta complejidad de la ciudad de Buenos Aires, se diagnosticaron 55 casos de 2016 a 2019 (comunicación personal).

Estos tumores se manifiestan con mayor frecuencia en pacientes con edades entre 6 y 9 años, pero se han reportado casos en niños menores de un año ${ }^{1,2}$. Se clasifican estos tumores de acuerdo con la histología, la localización, la infiltración y el patrón de progresión. Los tipos más frecuentes son el glioma difuso, el glioma exofítico dorsal, el glioma focal tectal y el glioma de la unión cervicomedular ${ }^{3}$.

El glioma difuso, o glioma pontino intrínseco difuso (DIPG, por sus siglas en inglés), se corresponde en la anatomía patológica con el astrocitoma anaplásico de alto grado (grado III en la clasificación de la Organización Mundial de la Salud) o con el glioblastoma multiforme (grado IV) ${ }^{4,5}$. El diagnóstico puede hacerse con resonancia magnética (RM), sobre todo con RM con espectroscopia, y en este caso se acepta que la información que ofrece no difiere del diagnóstico anatomopatológico ${ }^{6}$. Otra opción es la biopsia estereotáxica, un método controvertido en el campo de la neurocirugía y neuroncología?

El glioma difuso es el más frecuente de los tumores de tronco encefálico ( $80 \%$ de los casos) y se caracteriza por su rápida evolución, con enorme deterioro del paciente, sobrevida limitada y elevada mortalidad, independiente del tratamiento ${ }^{1,8}$. La media de sobrevida es de 10 meses, y el tratamiento con radioterapia puede mejorar transitoriamente los síntomas neurológicos y extender la sobrevida en 6 a 9 meses, no más9.

Los National Institutes of Health (NIH) señalan el valor de la resonancia magnética para definir la condición del tumor (difuso o focal) y explican que disponer de una muestra de biopsia permite el diagnóstico histológico y, además, la realización de pruebas inmunohistoquímicas, citogenéticas y de genética molecular, lo que favorece un diagnóstico más preciso y la clasificación de las neoplasias ${ }^{10}$. Los NIH indican la resección parcial y la biopsia para el diagnóstico de los tumores focales; para los tumores difusos, que habitualmente no eran sometidos a cirugía para biopsia, la agencia ahora recomienda técnicas de biopsia estereotáxica (más seguras e inocuas) con el objetivo de disponer de tejido para la caracterización molecular del tumor, siempre dentro del marco de los ensayos clínicos ${ }^{10}$.

En Argentina, el Ministerio de Salud publicó un documento de consenso de opinión de expertos sobre tumores del sistema nervioso central en niños. Con relación a los tumores de tronco, un $40 \%$ de los especialistas consultados sugiere hacer biopsia estereotáxica en tumores focales, y un $60 \%$ refiere hacerla "siempre que sea posible" ${ }^{11}$. El documento señala que la biopsia en el tumor difuso no es necesaria porque no cambia el tratamiento y tendría un carácter de investigación con fines académicos. En el futuro, cuando la inmunohistoquímica identifique alguna terapia target en particular, como es el caso de la histona mutada, tendrá utilidad la biopsia ${ }^{11}$.

Quick-Weller y colaboradores señalan el valor de la biopsia para disponer de una muestra de tejido que permite realizar, además del estudio anatomopatológico e inmunohistoquímico clásico, la evaluación molecular y el análisis del genoma, lo que permite categorizar mejor a estos tumores y ofrecer una terapia individualizada para cada paciente ${ }^{6}$. En el mismo sentido, la European Society for Medical Oncology y la American Society of Clinical Oncology también enfatizan el valor de la caracterización molecular y asocian la histona H3.1K27M con mayor sobrevida que la histona $\mathrm{H} 3.3 \mathrm{~K} 27 \mathrm{M}^{12}$.

Frente a este panorama es necesario analizar, en una profunda y honesta reflexión, la indicación de cirugía para estos tumores, sobre todo para el tumor difuso, ya sea con indicación de biopsia diagnóstica o con criterio de resección tumoral. Esta reflexión requiere, además de conocimientos técnico-quirúrgicos, un profundo análisis bioético con el objetivo de responder si es moral o no someter pacientes a un procedimiento quirúrgico teniendo en cuenta el mal pronóstico que presentan.

\section{Reflexión sobre la indicación quirúrgica}

En principio, cuando se propone un tratamiento a un paciente, el objetivo inicial es producir un 
beneficio o, por lo menos, no producir más daño que el ocasionado por la enfermedad en sí misma. En este caso, las decisiones sobre procedimientos quirúrgicos obligan a evaluar cuidadosamente los riesgos y beneficios de una cirugía ${ }^{13}$.

Cuando se analizan estos riesgos y beneficios, se deben tener en cuenta aspectos técnicos vinculados a la posibilidad de complicaciones propias de una cirugía, como hemorragias, infecciones, riesgo anestésico y alteraciones en la función respiratoria y cardíaca. Respecto a la biopsia estereotáxica de tumores del tronco encefálico, algunos autores mencionan un $1 \%$ de pacientes con déficit neurológico transitorio postquirúrgico y un $0,3 \%$ de mortalidad entre niños y adultos ${ }^{7}$, mientras otros señalan hasta un $9 \%$ de complicaciones en niños ${ }^{1}$.

En el caso particular de estos tumores, la toma de una muestra del tejido tumoral sirve para confirmar un diagnóstico anatomopatológico. Sin embargo, desde el advenimiento de la resonancia magnética, se puede definir con precisión la localización y las características del tumor (si focal o difuso) para tomar decisiones sobre la cirugía con mayor seguridad e inclusive decidir sobre el tratamiento con radioterapia. En la actualidad, además, se agrega la posibilidad de realizar estudios de inmunohistoquímica y análisis molecular con la muestra de tejido obtenida. Estos estudios permiten definir mejor el diagnóstico tumoral y abren posibilidades de pronóstico y tratamiento en el futuro.

Hoy la única opción de tratamiento, independiente del resultado de anatomía patológica, es la radioterapia, y siempre se debe tener en cuenta que el pronóstico es malo y la sobrevida es corta (sobre todo en los tumores difusos). En un extenso metanálisis, Hassan y colaboradore ${ }^{2}$ investigan los factores pronósticos y de sobrevida en niños con tumores del tronco encefálico y observan que no hay diferencias en la sobrevida cuando se analiza la clasificación tumoral, los diversos regímenes de radioterapia y el uso de temozolomida (aunque haya un amplio intervalo de confianza en estos datos). Además, los autores mencionan que aún no hay datos suficientes para sacar conclusiones con relación a la edad de los pacientes, la duración de los síntomas antes del diagnóstico y el hallazgo de mutación de histona K27M y mutación PCVR1 ${ }^{2}$.

Frente a esto y teniendo en cuenta los riesgos y el escaso beneficio del procedimiento, ¿la biopsia estereotáxica en niños se justifica? ¿Es justo someter a un niño a una cirugía que no le producirá beneficios significativos ni mejorará su calidad de vida?

En bioética hay un concepto muy arraigado, denominado "interés superior del niño", que puede y debe aplicarse en la toma de decisiones por parte del equipo clínico-quirúrgico. Garantizar este interés consiste en considerar al niño enfermo $-y$, por lo tanto, vulnerable - como objeto central de las decisiones. Ante todo, importa el bienestar del paciente y no agregar más daños. Este concepto del mejor interés está descripto en la Convención sobre los Derechos del Niño, que Argentina suscribió e incorporó a la Ley $26.061 / 2005$, la Ley de Protección Integral de los Derechos de los Niños, Niñas y Adolescentes ${ }^{14}$.

Sin dejar de considerar esta premisa y apelando a la responsabilidad del equipo médico-quirúrgico, surge la preocupación e interés de los profesionales de ofrecer alternativas para los niños con tumores del tronco encefálico. Por tal motivo, el estudio de marcadores moleculares e inmunohistoquímicos tumorales abre caminos para definir conductas terapéuticas e intentar mejorar el pronóstico y la calidad de vida de estos pacientes.

Sin embargo, se debe tener en cuenta que si se somete a un paciente a biopsia tumoral para detectar marcadores celulares en ese tejido, y considerando el conocimiento actual sobre el tema, es muy probable que esas informaciones no signifiquen un beneficio para ese niño en particular. Por tal razón, es muy importante el diálogo honesto con la familia del niño (y si es posible con el paciente) para acordar la conducta quirúrgica y no generar falsas expectativas sobre el resultado del procedimiento, que podrá ayudar a otros niños en el futuro, pero muy probablemente no ayudará al paciente. Es evidente que la aceptación para realizar este procedimiento estaría dada por motivos altruistas y mostraría una actitud solidaria hacia otros niños que padecerán la misma enfermedad, lo que puede ser emocionalmente positivo para el niño y su familia.

Se plantea un dilema con relación a esta actitud altruista, que genera diversas opiniones. Hay quien considera que participar en una investigación clínica es un deber moral, porque buenas investigaciones redundan en beneficio de toda la sociedad. Con base en este concepto, los niños y sus padres 
tendrían razones morales para aceptar realizar una biopsia tumoral con fines científicos. Por otro lado, otros consideran que, dada su condición de gravedad y vulnerabilidad, estos niños deben ser protegidos de todo procedimiento invasivo.

Dado que hay opiniones fuertes y contradictorias a favor y en contra de la biopsia, es difícil responder si este procedimiento respeta el mejor interés del niño ${ }^{15}$. En este caso es crucial la actitud del médico neurocirujano, del oncopediatra y del pediatra clínico que intervienen en las decisiones. Estos profesionales deben ser sinceros con la familia del niño y disponer de tiempo para dialogar honestamente y ofrecer informaciones precisas sobre la enfermedad, sus características, pronóstico y tratamiento, sobre todo en relación con la indicación de cirugía, que muchas veces es vista por los padres angustiados como una solución.

En estas circunstancias, el equipo médico debe evitar caer en una actitud paternalista. Toda la información que se transmite al paciente y su familia debe ser plasmada por escrito en el documento de consentimiento informado, donde se señalarán riesgos y beneficios de la cirugía, si el procedimiento es parte o no de un ensayo clínico y si el material de biopsia será resguardado en el hospital para futuros estudios.

Sin duda, quedará a criterio del equipo médico decidir cuándo intervenir, con base en criterios profesionales y en el análisis de cada caso en particular. El procedimiento tal vez se justifique en niños con condiciones clínicas neurológicas razonables, y siempre con el acuerdo de los padres o encargados del paciente, quienes son los que representan al menor y deben ejercer plenamente la autonomía ${ }^{16}$ en la toma de decisiones basadas en la comprensión de la información recibida. Cabe señalar que en los niños la autonomía es progresiva de acuerdo con su maduración y desarrollo, y que estos pacientes deben recibir las informaciones sobre el procedimiento a que serán sometidos en la medida de su capacidad de comprensión ${ }^{17}$.

En esta situación, como en tantas otras, el avance de la ciencia y de la tecnología trae desafíos, y para responder a ellos se requieren no solo decisiones científicas, sino también analizar los valores morales en juego. La ética es una herramienta que puede ayudar a reflexionar sobre estos valores. Sin duda, la ciencia debe buscar nuevas opciones de diagnóstico y tratamiento por el bien de los seres humanos, pero siempre con responsabilidad. En este sentido, es importante resaltar el comité de ética clínica como ámbito más adecuado para un análisis reflexivo interdisciplinario.

Las decisiones relativas a niños con tumores del tronco encefálico deben ser cuidadosamente evaluadas por un equipo médico quirúrgico, y no individualmente. A su vez, la incorporación de estos niños en ensayos clínicos debe ser evaluada por comités de ética en investigación. Este es un modo de incorporar diferentes miradas y opiniones, siempre con el objetivo de ofrecer lo mejor al paciente, pensando en su interés y en su bienestar. Es justamente el comité de ética de investigación quien debe proteger a los niños que, además de pacientes, son sujetos de investigación.

\section{Consideraciones finales}

Las investigaciones científicas traen beneficios para la sociedad y las futuras generaciones, y se tienen en cuenta estos beneficios cuando se incluyen niños en investigaciones que no los beneficiarán directamente, pues ellos son los únicos que podrán ayudar a otros niños en condiciones similares ${ }^{18}$. Sin embargo, aunque hay que reconocer el enorme valor de las investigaciones, siempre se debe considerar las obligaciones de todas las personas o entidades que intervienen en un protocolo de ensayo clínico (en el caso aquí analizado, neurocirujanos, médicos anatomopatólogos, neuroncólogos, pediatras clínicos, autoridades del hospital y comités de ética en investigación).

De acuerdo con Jonas ${ }^{18}$, la tecnología no es neutra y puede emplearse tanto para el bien como para el mal. Ella genera poder y, por lo tanto, exige responsabilidad en quien la utiliza. Siempre es el bien humano que se debe promover, respetando los intereses y derechos de las personas, reparando injusticias y aliviando padecimientos. El objeto de la obligación humana son los hombres ${ }^{18}$.

Los tumores de tronco constituyen un ejemplo claro en el que el equipo médico quirúrgico debe garantizar el respeto a la autonomía del paciente y su familia, conciliando la práctica asistencial, que tiene un objetivo definido de beneficencia, con la investigación clínica, que no siempre aporta un beneficio concreto para el paciente. 


\section{Referencias}

1. Laigle-Donadey F, Doz F, Delattre JY. Brainstem tumors. In: Grisold W, Soffietti R, editores. Handbook of clinical neurology [Internet]. Amsterdam: Elsevier; 2012 [acesso 10 fev 2021]. p. 585-605. DOI: 10.1016/ B978-0-444-53502-3.00010-0

2. Hassan H, Pinches A, Picton SV, Phillips R. Survival rates and prognostic predictors of high grade brain stem gliomas in childhood: a systematic review and meta-analysis. J Neurooncol [Internet]. 2017 [acesso $10 \mathrm{fev}$ 2021];135:13-20. DOI: 10.1007/s11060-017-2546-1

3. Guillamo JS, Doz F, Delattre JY. Brain stem gliomas. Curr Opin Neurol [Internet]. 2001 [acesso 10 fev 2021];14(6):711-5. DOI: 10.1097/00019052-200112000-00006

4. Lin TF, Prados M. Brainstem gliomas. In: Gupta N, Banerjee A, Haas-Kogan DA, editores. Pediatric CNS tumors. $3^{\text {a }}$ ed. Berlin: Springer; 2017. p. 49-65.

5. Saratsis AM, Nazarian J, Magge SN. Brainstem gliomas. In: Keating R, Goodrich JT, Packer R, editores. Tumors of the pediatric central nervous system. $2^{\text {a }}$ ed. New York: Thieme; 2013. p. 347-53.

6. Quick-Weller J, Tritt S, Behmanesh B, Mittelbronn M, Spyrantis A, Dinc C et al. Biopsies of pediatric brainstem lesions display low morbidity but strong impact on further treatment decisions. J Clin Neurosci [Internet]. 2017 [acesso 10 fev 2021];44:254-9. DOI: 10.1016/j.jocn.2017.06.028

7. Leach PA, Listlin EJ, Coope DJ, Thorne JA, Kamaly-AsI ID, The Royal Manchester Children's Hospital Clinical Neuroscience Group. Diffuse brainstem gliomas in children: should we or shouldn't we biopsy? Br J Neurosurg [Internet]. 2008 [acesso 10 fev 2020];22(5):619-24. DOI: 10.1080/02688690802366198

8. Souza P, Hinojosa J, Muñoz MJ, Esparza J, Muñoz A. Gliomas del tronco encefálico. Neurocirugía [Internet]. 2004 [acesso 10 fev 2020];15(1):56-66. DOI: 10.1016/S1130-1473(04)70502-3

9. Maxwell R, Luksik AS, Garzon-Muvdi T, Yang W, Huang J, Bettegowda C et al. Population-based study determining predictors of cancer-specific mortality and survival in pediatric high-grade brainstem glioma. World Neurosurg [Internet]. 2018 [acesso 10 fev 2020];119:1006-15. DOI: 10.1016/j.wneu.2018.08.044

10. National Cancer Institute. Childhood brain stem glioma treatment ( $\mathrm{PDQ}^{\oplus}$ ): health professional version [Internet]. 2020 [acesso $10 \mathrm{fev}$ 2020]. Disponível: https://bit.ly/37Hr9B1

11. Fischman M, Ismael J, Pesce P, Rufach D. Tumores pediátricos del sistema nervioso central [Internet]. Buenos Aires: Ministerio de Salud; 2015 [acesso 10 fev 2020]. Disponível: https://bit.ly/3iLTQ69

12. Hoffman L, Veldhuijzen van Zanten SEM, Colditz N, Baugh J, Chaney B, Hoffmann M et al. Clinical, radiologic, pathologic, and molecular characteristics of long-term survivors of diffuse intrinsic pontine glioma (DIPG): a collaborative report from the International and European Society for Pediatric Oncology DIPG registries. J Clin Oncol [Internet]. 2018 [acesso 10 fev 2020];36(19):1963-72. DOI: 10.1200/JCO.2017.75.9308

13. Luna F. Planteos clásicos y teoría de los principios. In: Luna F, Salles A. Bioética: nuevas reflexiones sobre debates clásicos. Buenos Aires: Fondo de Cultura Económica; 2008. p. 23-78

14. Argentina. Ley $n^{\circ}$ 26.061. Protección integral de los derechos de niñas, niños y adolescentes [Internet]. Buenos Aires: Ministerio de Justicia y Derechos Humanos de la Nación; 2014 [acesso 10 fev 2020]. Disponível: https://bit.ly/3iNKLtQ

15. Wilkinson R, Harris J. Razões morais e legais para o altruísmo no caso de biópsia do tronco cerebral em glioma difuso. Br J Neurosurg [Internet]. 2008 [acesso $10 \mathrm{fev}$ 2020];22(5):617-18. DOI: $10.1080 / 02688690802482896$

16. Beauchamp T, Childress J. Principios de ética biomédica. $4^{\text {a }}$ ed. Barcelona: Masson; 1999.

17. Ciruzzi MS. La competencia bioética en el menor de edad. In: Ciruzzi MS. La autonomia del paciente pediátrico: ¿mito, utopía o realidad? Buenos Aires: Cathedra Jurídica; 2011. p. 37-77.

18. Jonas H. Técnica, medicina y ética: la práctica del principio de responsabilidad. Barcelona: Paidós; 1997. 
Adriana Scrigni - Magíster - ascrigni@intramed.net

(iD) 0000-0001-7389-2933

Beatriz Mantese - Graduada - bmantese@yahoo.com.ar

(D) 0000-0001-9575-7759

\section{Correspondencia}

Adriana Scrigni - Tucumán 1657, 8. Depto. A C 1050. Buenos Aires, Argentina.

Participación de las autoras

Adriana Scrigni escribió el artículo. Beatriz Mantese contribuyó con la indicación de referencias bibliográficas y con la revisión del escrito.

Recibido: 19.8 .2020

Revisado: $\quad 3.8 .2021$

Aprobado: $\quad 5.8 .2021$ 